\title{
Delivery of Small Interfering RNA for Inhibition of Endothelial Cell Apoptosis by Hypoxia and Serum Deprivation
}

\author{
Seung-Woo Cho' ${ }^{1}$ Lauren Hartle ${ }^{2}$, Sun Mi Son ${ }^{1}$, Fan Yang ${ }^{1}$, Michael Goldberg ${ }^{3}$, Qiaobing \\ $\mathbf{X u}^{1}$, Robert Langer ${ }^{1,3,4}$, and Daniel G. Anderson ${ }^{4,}{ }^{\star}$ \\ ${ }^{1}$ Department of Chemical Engineering, Massachusetts Institute of Technology, Cambridge, MA \\ 02139, USA \\ ${ }^{2}$ Department of Physics and Astronomy, University of North Carolina, Chapel Hill, NC 27599, \\ USA
}

${ }^{3}$ Department of Chemistry, Massachusetts Institute of Technology, Cambridge, MA 02139, USA

${ }^{4}$ David H. Koch Institute for Integrative Cancer Research, Massachusetts Institute of Technology, Cambridge, MA 02139, USA

\begin{abstract}
RNA interference (RNAi) for anti-angiogenic or pro-apoptotic factors in endothelial cells (ECs) has great potential for the treatment of ischemic diseases by promoting angiogenesis or inhibiting apoptosis. Here, we report the utility of small interfering RNA (siRNA) in inhibiting EC apoptosis induced by tumor necrosis factor- $a$ (TNF- $\alpha$ ). siRNA was designed and synthesized targeting tumor necrosis factor-a receptor-1 (TNFR-1) and Src homology 2 domain-containing protein tyrosine phosphatase-1 (SHP-1). Human umbilical vein endothelial cells (HUVECs) were cultured under in vitro hypoxic and serum-deprived conditions to simulate in vivo ischemic conditions. Two days after liposomal delivery of siRNA targeting TNFR-1 and SHP-1, significant silencing of each target (TNFR-1; $76.5 \%$ and SHP-1; $97.2 \%$ ) was detected. Under serum-deprived hypoxic ( $1 \%$ oxygen) conditions, TNF-a expression in HUVECs increased relative to normoxic (20\% oxygen) and serum-containing conditions. Despite enhanced TNF-a expression, suppression of TNFR-1 or SHP-1 by siRNA delivery not only enhanced expression of angiogenic factors (KDR/ Flk-1 and eNOS) and anti-apoptotic factor (Bcl-xL) but also reduced expression of a pro-apoptotic factor (Bax). Transfection of TNFR-1 or SHP-1 siRNA significantly decreased the HUVEC apoptosis while significantly enhancing HUVEC proliferation and capillary formation. The present study demonstrates that TNFR-1 and SHP-1 may be useful targets for the treatment of myocardial or hindlimb ischemia.
\end{abstract}

\section{Keywords}

RNA interference; Endothelial cell; Hypoxia; Serum Deprivation; Apoptosis

\footnotetext{
(C) 2008 Elsevier Inc. All rights reserved.

*Corresponding author: Daniel G. Anderson, Ph.D., David H. Koch Institute for Integrative Cancer Research, Massachusetts Institute of Technology, 45 Carleton Street, E25-342, Cambridge, MA 02139, USA, Tel.: 617-258-6843; Fax: 617-258-8827; dgander@mit.edu.

Publisher's Disclaimer: This is a PDF file of an unedited manuscript that has been accepted for publication. As a service to our customers we are providing this early version of the manuscript. The manuscript will undergo copyediting, typesetting, and review of the resulting proof before it is published in its final citable form. Please note that during the production process errors may be discovered which could affect the content, and all legal disclaimers that apply to the journal pertain.
} 


\section{Introduction}

Ischemia is associated with apoptosis of endothelial cells (ECs) due to hypoxia and deficiency of survival growth factors [1-4]. During the ischemic event, tumor necrosis factor- $\alpha$ (TNF- $\alpha$ ) induces EC apoptosis, resulting in impaired angiogenesis [5,6]. Several molecules are known to mediate EC apoptosis induced by TNF-a, including Src homology 2 domain-containing protein tyrosine phosphatase-1 (SHP-1) [5,6] and tumor necrosis factora receptor-1 (TNFR-1) [7]. SHP-1 activated by TNF-a can inhibit EC proliferation and angiogenesis via the inactivation of vascular endothelial growth factor (VEGF) receptor-2 (KDR/Flk-1) in ECs [5,6,8,9]. In addition to its antiangiogenic activity, SHP-1 also plays a significant role in apoptosis in many cell types [10-12]. Previous studies have reported upregulation of SHP-1 expression following myocardial or cerebral ischemia, which may contribute to the increased myocardial [12] or cerebral [13] infarction. TNFR-1 is also stimulated by TNF-a binding and negatively modulates angiogenesis through the inactivation of KDR/Flk-1 and subsequent inhibition of EC proliferation [7,14,15]. TNFR-1 activation by TNF-a upregulated after myocardial or hindlimb ischemia impairs angiogenesis and contributes to tissue apoptosis and the consequent progression of ischemic diseases $[14,15]$.

We hypothesize that the specific silencing of gene expression using RNA interference (RNAi) could be used to interrupt anti-angiogenic and apoptotic signal pathways mediated by SHP-1 or TNFR-1. Silencing of SHP-1 or TNFR-1 expression with small interfering RNA (siRNA) may enhance the proliferative and angiogenic capacity of ECs and inhibit apoptosis of ECs under in vitro and in vivo ischemic conditions, thereby facilitating rescue of damaged tissues. Here, we examine the potential of siRNA targeting SHP-1 or TNFR-1 to improve the proliferative and angiogenic properties of human umbilical vein endothelial cells (HUVECs) under hypoxic and serum-deprived conditions, a simulated ischemic condition.

\section{Materials and Methods SiRNAs}

All siRNAs were synthesized by Dharmacon (Lafayette, CO, USA). Two siRNAs were designed for targeting human TNFR-1 (GenBank accession number: NM_001065) and human SHP-1 (GenBank accession number: NM_080548). Green Fluorescent Protein (GFP) siRNA was used as a negative control [16]. The sequences for the sense and anti-sense strands of siRNAs are: 1) TNFR-1, (sense) 5'-CAAAGG AAC CUA CUU GUA CUU-3', (anti-sense) 5'-GUA CAA GUA GGU UCC UUU GUU-3', 2) SHP-1, (sense) 5'GGAACAAAU GCG UCC CAU AUU-3', (anti-sense) 5'-UAU GGG ACG CAU UUG UUC CUU-3', 3) GFP, (sense) 5'-CAA GCU GAC CCU GAA GUU CUU-3', (anti-sense) 5'-GAA CUU CAG GGU CAG CUU GUG-3'. All siRNAs were generated by annealing equimolar amounts of complementary sense and anti-sense strands.

\section{SiRNA transfection}

HUVECs (Cambrex, Walkersville, MD, USA) were cultured in EGM-2 medium (Cambrex) supplemented with the SingleQuot kit at $37{ }^{\circ} \mathrm{C}$ and $5 \% \mathrm{CO}_{2}$. HUVECs at passage three were used for transfection. For examination of cell viability and apoptosis, HUVECs were seeded (10,000 cells per well) into 96-well polystyrene plates (Corning-Costar, Kennebunk, ME, USA) and allowed to attach overnight in EGM-2 medium. HUVECs were transfected with siRNAs complexed with Lipofectamine ${ }^{\mathrm{TM}} 2000(1.0 \mathrm{mg} / \mathrm{ml}$, Invitrogen, Carlsbad, CA, USA) as described by the vendor. Briefly, Lipofectamine diluted in serum-free Opti-MEM (Invitrogen) was added to the siRNAs premixed with Opti-MEM at a 2.5:1 Lipofectamine- 
to-siRNA weight-weight ratio, and the mixtures were incubated for $20 \mathrm{~min}$ at room temperature to allow for complex formation. The mixtures were added (50 ng of siRNA per well) to EGM-2 medium in each well of the 96-well polystyrene plates. Growth medium was removed from the cells, and the EGM-2 medium containing Lipofectamine/siRNA complexes was immediately transferred to the cells. Transfection was performed in quadruplicate. For reverse transcription-polymerase chain reaction (RT-PCR) and real-time PCR, HUVECs seeded into each well of 6-well polystyrene plates $\left(2.0 \times 10^{5}\right.$ cells per well $)$ were transfected with siRNAs complexed with Lipofectamine at $2.5(\mathrm{w} / \mathrm{w})$ ratio of Lipofectamine to siRNA ( $1 \mu \mathrm{g}$ of siRNA per well). After transfection, cells were allowed to grow for two days at $37{ }^{\circ} \mathrm{C}$ and $5 \% \mathrm{CO}_{2}$. Transfection efficiency was determined by measuring glyceraldehyde-3-phosphate dehydrogenase (GAPDH) activity in HUVECs 2 days after transfection with human GAPDH siRNA (Ambion, Austin, TX, USA). GAPDH activity was measured using the KDalert ${ }^{\mathrm{TM}}$ GAPDH assay kit (Ambion) according to the manufacture's instructions. GAPDH activity of GAPDH siRNA-transfected HUVECs was expressed as a percent activity to that of GFP siRNA-transfected HUVECs.

\section{HUVEC culture under hypoxic and serum-free conditions}

Two days after transfection with SHP-1, TNFR-1, or GFP siRNA, HUVECs were cultured under hypoxic and serum-free conditions. EGM-2 medium was changed to EBM-2 (endothelial basal medium) without fetal bovine serum and growth factors, and siRNAtransfected HUVECs were further cultured for one or two days in a hypoxic incubator (MCO-18M, Sanyo, Japan) with $1 \%$ oxygen and $5 \% \mathrm{CO}_{2}$ at $37^{\circ} \mathrm{C}$. Low oxygen content was maintained through the controlled supply of nitrogen gas to the incubator.

\section{RT-PCR}

Gene expression profiles in siRNA-transfected HUVECs after culture under hypoxic and serum-deprived conditions were examined with RT-PCR. Total RNA was isolated using the RNeasy Mini kit (Qiagen, Chatsworth, CA, USA) from each sample of cells ( $\mathrm{n}=4$ per group). The RNA concentration was determined by measuring absorbance at $260 \mathrm{~nm}$ using a spectrophotometer. A reverse transcription reaction was performed with $1 \mu \mathrm{g}$ pure total RNA using SuperScript ${ }^{\mathrm{TM}}$ III reverse transcriptase (Invitrogen). The synthesized cDNA was amplified by PCR with Platinum PCR Master Mix (Invitrogen). The sequences and product sizes of human-specific primers are listed in Table 1. The amplification conditions followed several steps: $5 \mathrm{~min}$ at $95^{\circ} \mathrm{C}$, followed by $25-35$ cycles of denaturing $\left(94^{\circ} \mathrm{C}, 30 \mathrm{sec}\right)$, annealing $\left(55-62^{\circ} \mathrm{C}, 30 \mathrm{sec}\right)$, and extension $\left(72^{\circ} \mathrm{C}, 45 \mathrm{sec}\right)$ with a final extension at $72^{\circ} \mathrm{C}$ for $7 \mathrm{~min}$. The PCR products were visualized by electrophoresis on a $2 \%$ agarose gel with ethidium bromide (E-Gel, Invitrogen).

\section{Quantitative real-time PCR (TaqMan method)}

Quantitative real-time PCR was performed using a 7500 Fast Real-Time PCR System (Applied Biosystems, Foster City, CA, USA). Universe Fast PCR Master Mix (Applied Biosystems) was used for PCR. Quantification of gene expression in siRNAtransfected HUVECs was performed with TaqMan ${ }^{\circledR}$ Gene Expression Assays (Applied Biosystems) for each target (TNFR-1: Hs00533568_g1, SHP-1: Hs00169359_m1, TNF-a: Hs00174128_m1, VEGF: Hs00173626_m1, KDR/Flk-1: Hs00176676_m1, eNOS: Hs00167166_m1, Bcl-xL: Hs00169141_m1, Bax: Hs00180269_m1, and GAPDH: Hs02758991_g1). The expression level of target genes was determined by the comparative $C_{t}$ method, whereby the target is normalized to the endogenous reference (GAPDH). The $C_{t}$ value is the cycle number at which the fluorescence level reaches its threshold. The $\Delta C_{t}$ was determined by subtracting the $C_{t}$ of GAPDH control from the $C_{t}$ of target gene $\left[\Delta C_{t}=C_{t}\right.$ (target) $\left.-C_{t}(\mathrm{GAPDH})\right]$. The relative value of target to endogenous reference was described as the fold of GAPDH $=$ 
$2^{-\Delta C t}$ [17]. The relative expression of TNFR-1 or SHP-1 siRNA-transfected HUVECs was normalized to that in GFP siRNA-transfected HUVECs.

\section{Determination of apoptotic activity}

One and two days after culture under hypoxic and serum-free conditions, the extent of apoptosis of siRNA-transfected HUVECs was determined by the terminal deoxynucleotide transferase-mediated deoxyuridine triphosphate nick end-labeling (TUNEL) method using a commercially-available apoptosis detection kit (ApopTaq ${ }^{\circledR}$, Chemicon, Temecula, CA, USA). Cells stained with rhodamine (red) by a TUNEL staining method were counterstained with 4,6-diamidino-2-phenylindole (DAPI, Vector Laboratories, Burlingame, CA, USA). The stained images were examined with a fluorescence microscope (Carl Zeiss, Oberkochen, Germany). The portion of TUNEL-positive cells among the total cell population was calculated as the percentage ratio of TUNEL-stained cells to DAPI-stained cells.

\section{Cell viability measurement}

Cell viability was measured using the CellTiter $96^{\circledR}$ Aqueous One Solution assay kit (Promega, Madison, WI, USA). Cellular metabolic activity was determined by measuring optical absorbance at $490 \mathrm{~nm}$ using a Victor3 Multilabel plate counter (Perkin-Elmer Life Sciences, Boston, MA, USA). The viability of siRNA-transfected HUVECs cultured under hypoxic (1\% oxygen) and serum-free (EBM-2 medium) conditions for one or two days was converted to percent viability by comparison to that of HUVECs cultured under normal condition with normoxia (20\% oxygen) and EGM-2 medium for the comparable time period.

\section{Capillary formation assay}

48-well polystyrene plates were coated with Growth Factor Reduced Matrigel (GFRMatrigel, BD Biosciences, San Jose, CA, USA). Two days after transfection, siRNAtransfected HUVECs were detached with $0.05 \%$ (w/v) trypsin, suspended in EBM-2 basal medium, and seeded at $4.0 \times 10^{4} / \mathrm{cm}^{2}$ onto GFR-Matrigel-coated plates. Cells were incubated under hypoxic (1\% oxygen) and serum-deprived (EBM-2 medium) conditions at $37^{\circ} \mathrm{C}$ and $5 \% \mathrm{CO}_{2}$. After two days of culture, the formation of capillary structures by HUVECs was examined microscopically.

\section{Statistical analysis}

Quantitative data are expressed as mean \pm standard deviation. Statistical analysis was performed by the unpaired Student's $t$ test using InStat software (InStat 3.0, GraphPad Software Inc., San Diego, CA, USA). A value of $\mathrm{p}<0.05$ was considered to be statistically significant.

\section{Results and Discussion}

Delivery of siRNA targeting TNFR-1 or SHP-1 specifically silences each target in HUVECs. In a preliminary transfection with GAPDH siRNA, 80\% reduction of GAPDH activity was observed in HUVECs two days after transfection. RT-PCR was performed to confirm the silencing of each molecule in HUVECs two days after siRNA transfection (Fig. 1A). The mRNA expression of TNFR-1 or SHP-1 was significantly reduced in HUVECs transfected with each siRNA compared to GFP siRNA-transfected or untransfected HUVECs (Fig. 1A). Quantitative real-time PCR analysis verified that groups treated with TNFR-1 or SHP-1 delivery resulted in $76.5 \%$ and $97.2 \%$ silencing, respectively, of each target molecule relative to the control group transfected with GFP siRNA (Fig. 1B and C). 
Under hypoxic (1\% oxygen) and serum-deprived conditions, silencing of TNFR-1 or SHP-1 expression led to an increase in the expression of angiogenic and anti-apoptotic factors and a decrease in the expression of pro-apoptotic factor in HUVECs. Hypoxia and serumdeprivation enhanced TNF- $a$ and VEGF expression in HUVECs (Fig. 2A). The expressions of TNF- $a$ and VEGF in HUVECs transfected with TNFR-1 or SHP-1 siRNA were not significantly different ( $p>0.05$ ) from those in HUVECs transfected with GFP siRNA (Fig. $2 \mathrm{~B}$ and $\mathrm{C}$ ). Despite enhanced expression of TNF- $\mathrm{a}$, which is known to downregulate KDR/ Flk-1 and eNOS expression in ECs [5,18,19], the expression levels of KDR/Flk-1 and eNOS were enhanced in HUVECs with TNFR-1 or SHP-1 siRNA transfection at day one and two after culture in the serum-deprived hypoxic condition (Fig. 2A, D, and E). Previous studies have shown that suppression of TNFR-1 or SHP-1 might increase KDR/Flk-1 expression [5,15]. Consequently, enhanced KDR/Flk-1 expression could increase eNOS expression [20]. The expression of the anti-apoptotic factor (Bcl-xL) were enhanced by silencing of TNFR-1 or SHP-1 (Fig. 2A and F), whereas the expression of the pro-apoptotic factor (Bax) was reduced in TNFR-1 or SHP-1 siRNA-transfected HUVECs compared with GFP siRNAtransfected HUVECs (Fig. 2A and G). These results are consistent with a previous report demonstrating that knockdown of SHP-1 by siRNA reversed the inhibition of STAT3 activation, thereby increasing the expression of STAT3-regulated gene products such as Bcl-2 and Bcl-xL [21]. SHP-1 has a direct effect on apoptosis in many cell types [10-12] by binding to death receptors such as TNFR-1 and FAS-R and by blocking anti-apoptotic signals [12,22]. Because TNFR-1 is also required to induce TNF-a -mediated apoptosis in ECs, knockdown of TNFR-1 may inhibit EC apoptosis by neutralizing TNF-a bioactivity [23]. Therefore, the decrease in Bax expression might result from reduction in apoptotic signals in ECs by silencing of TNFR-1 or SHP-1.

Apoptosis of HUVECs cultured under hypoxic and serum-free conditions was significantly reduced by silencing TNFR-1 or SHP-1 expression. Favorable changes in gene expression profiles induced by TNFR-1 or SHP-1 siRNA delivery (Fig. 2) may inhibit EC apoptosis and enhance EC proliferation. A cell apoptosis assay (TUNEL staining) at day two after culture showed that apoptosis was more extensive in GFP siRNA-transfected HUVECs than in TNFR-1 or SHP-1 siRNA-transfected HUVECs (Fig. 3A). The portion of TUNELpositive cells was much higher in HUVECs with GFP siRNA than in HUVECs with TNFR-1 or SHP-1 siRNA ( $<0.01$, compared to GFP siRNA, Fig. 3B). Additionally, TNFR-1 or SHP-1 siRNA-transfected HUVECs showed greater cell proliferation than GFP siRNA-transfected HUVECs at days one and two after culture under hypoxic (1\% oxygen) and serum-deprived conditions ( $\mathrm{p}<0.01$, compared to GFP siRNA, Fig. $3 \mathrm{C}$ ). In vitro culture conditions with low oxygen content and lack of survival growth factors mimic the microenvironment in ischemic tissues. We hypothesize that in vivo delivery of siRNA for TNFR-1 or SHP-1 may have utility for the treatment of ischemia via their angiogenic and/or anti-apoptotic effects on ECs. Of note, the administration of soluble TNFR-1 gene as an antagonist to TNF-a to interrupt signal pathways dependent on TNF-a has been reported to increase angiogenesis in ischemic tissues $[14,15]$. Injection of plasmid vector-based siRNA targeting SHP-1 also significantly reduced cardiomyocyte apoptosis and the infarcted area in ischemic myocardium [12].

The administration of siRNA targeting TNFR-1 or SHP-1 stimulated capillary formation by HUVECs under hypoxic and serum-deprived conditions. When cultured onto GFR-Matrigel, TNFR-1 or SHP-1 siRNA-transfected HUVECs showed more extensive capillary formation compared to GFP siRNA-transfected HUVECs (Fig. 4A-C). The number of lumen and tubes formed by HUVECs was significantly higher in TNFR-1 or SHP-1 siRNA group than in GFP siRNA group (Fig. 4D and E). This result suggests that the capacity of ECs inducing angiogenesis under ischemic conditions could be enhanced by silencing molecules with antiangiogenic effects, such as TNFR-1 and SHP-1. TNF-a mediates association of SHP-1 with 
$\mathrm{KDR} / \mathrm{Flk}-1$, which is reported to negatively regulate VEGF-mediated signal transduction [5,6]. TNFR-1 activation by TNF- $a$ also inhibits EC proliferation by VEGF signaling [15]. In the context of engineered cell therapy, angiogenic cells genetically modified with siRNAs for anti-angiogenic factors ex vivo might afford a higher therapeutic efficacy than cells without siRNA modification as a cell therapy source for ischemic disease treatment.

RNAi targeting for anti-angiogenic or pro-apoptotic factors may provide an alternative therapy for treatment of ischemia. The target molecules tested in the present study would be good candidates for RNAi therapeutics for ischemic diseases. Exploration of potential targets that are involved in more critical signaling in ischemic pathology (e.g. PHD1, an oxygen sensor that regulates the stability of the hypoxia-inducible factors under ischemia [24]) should be performed together with the development of efficient delivery vehicles for systemic or local siRNA delivery to ischemic tissues.

\section{Acknowledgments}

This work was supported by a grant (EB000244) from the National Institutes of Health. The authors also thank the support of "Alnylam Pharmaceuticals under Agmt. Dtd. 5/8/07". S.-W.C. would like to thank the Korea Medical Institution for his postdoctoral fellowship.

\section{References}

1. Lee CN, Cheng WF, Chang MC, Su YN, Chen CA, Hsieh FJ. Hypoxia-induced apoptosis in endothelial cells and embryonic stem cells. Apoptosis. 2005; 10:887-894. [PubMed: 16133878]

2. Hogg N, Browning J, Howard T, Winterford C, Fitzpatrick D, Gobé G. Apoptosis in vascular endothelial cells caused by serum deprivation, oxidative stress and transforming growth factor-beta. Endothelium. 1999; 7:35-49. [PubMed: 10599559]

3. Matsushita H, Morishita R, Nata T, Aoki M, Nakagami H, Taniyama Y, Yamamoto K, Higaki J, Yasufumi K, Ogihara T. Hypoxia-induced endothelial apoptosis through nuclear factor-kappaB (NF-kappaB)-mediated bcl-2 suppression: in vivo evidence of the importance of NF-kappaB in endothelial cell regulation. Circ. Res. 2000; 86:974-981. [PubMed: 10807870]

4. Meeson AP, Argilla M, Ko K, Witte L, Lang RA. VEGF deprivation-induced apoptosis is a component of programmed capillary regression. Development. 1999; 126:1407-1415. [PubMed: 10068634]

5. Nakagami H, Cui TX, Iwai M, Shiuchi T, Takeda-Matsubara Y, Wu L, Horiuchi M. Tumor necrosis factor-a inhibits growth factor-mediated cell proliferation through SHP-1 activation in endothelial cells. Arterioscler. Thromb. Vasc. Biol. 2002; 22:238-242. [PubMed: 11834522]

6. Guo DQ, Wu LW, Dunbar JD, Ozes ON, Mayo LD, Kessler KM, Gustin JA, Baerwald MR, Jaffe EA, Warren RS, Donner DB. Tumor necrosis factor employs a proteintyrosine phosphatase to inhibit activation of KDR and vascular endothelial cell growth factor-induced endothelial cell proliferation. J. Biol. Chem. 2000; 275:11216-11221. [PubMed: 10753929]

7. Sugano M, Tsuchida K, Tomita H, Makino N. Increased proliferation of endothelial cells with overexpression of soluble TNF-alpha receptor I gene. Atherosclerosis. 2002; 162:77-84. [PubMed: 11947900]

8. Seo DW, Li H, Qu CK, Oh J, Kim YS, Diaz T, Wei B, Han JW, Stetler-Stevenson WG. Shp-1 mediates the antiproliferative activity of tissue inhibitor of metalloproteinase-2 in human microvascular endothelial cells. J. Biol. Chem. 2006; 281:3711-3721. [PubMed: 16326706]

9. Bhattacharya R, Kwon J, Wang E, Mukherjee P, Mukhopadhyay D. Src homology 2 (SH2) domain containing protein tyrosine phosphatase-1 (SHP-1) dephosphorylates VEGF Receptor-2 and attenuates endothelial DNA synthesis, but not migration. J. Mol. Signal. 2008; 3:8. [PubMed: 18377662]

10. Mizuno K, Tagawa Y, Mitomo K, Watanabe N, Katagiri T, Ogimoto M, Yakura H. Src homology region 2 domain-containing phosphatase 1 positively regulates $\mathrm{B}$ cell receptor-induced apoptosis by modulating association of B cell linker protein with Nck and activation of c-Jun NH2-terminal kinase. J. Immunol. 2000; 169:778-786. [PubMed: 12097380] 
11. Thangaraju M, Sharma K, Leber B, Andrews DW, Shen SH, Srikant CB. Regulation of acidification and apoptosis by SHP-1 and Bcl-2. J. Biol. Chem. 1999; 274:29549-29557. [PubMed: 10506221]

12. Sugano M, Tsuchida K, Hata T, Makino N. RNA interference targeting SHP-1 attenuates myocardial infarction in rats. FASEB J. 2005; 19:2054-2056. [PubMed: 16223786]

13. Wishcamper CA, Brooks DM, Douglas Coffin J, Lurie DI. Focal cerebral ischemia upregulates SHP-1 in reactive astrocytes in juvenile mice. Brain Res. 2003; 974:88-98. [PubMed: 12742627]

14. Sugano M, Koyanagi M, Tsuchida K, Hata T, Makino N. In vivo gene transfer of soluble TNFalpha receptor 1 alleviates myocardial infarction. FASEB J. 2002; 16:1421-1422. [PubMed: 12205034]

15. Sugano M, Tsuchida K, Makino N. Intramuscular gene transfer of soluble tumor necrosis factoralpha receptor 1 activates vascular endothelial growth factor receptor and accelerates angiogenesis in a rat model of hindlimb ischemia. Circulation. 2004; 109:797-802. [PubMed: 14970118]

16. Krichevsky AM, Kosik KS. RNAi functions in cultured mammalian neurons. Proc. Natl. Acad. Sci. USA. 2002; 99:11926-11929. [PubMed: 12192088]

17. Kim J, Moon SH, Lee SH, Lee DR, Koh GY, Chung HM. Effective isolation and culture of endothelial cells in embryoid body differentiated from human embryonic stem cells. Stem Cells Dev. 2007; 16:269-280. [PubMed: 17521238]

18. Lai PF, Mohamed F, Monge JC, Stewart DJ. Downregulation of eNOS mRNA expression by TNFalpha: identification and functional characterization of RNA-protein interactions in the 3'UTR. Cardiovasc. Res. 2003; 59:160-168. [PubMed: 12829187]

19. Anderson HD, Rahmutula D, Gardner DG. Tumor necrosis factor-alpha inhibits endothelial nitricoxide synthase gene promoter activity in bovine aortic endothelial cells. J. Biol. Chem. 2004; 279:963-969. [PubMed: 14581470]

20. Shen BQ, Lee DY, Zioncheck TF. Vascular endothelial growth factor governs endothelial nitricoxide synthase expression via a KDR/Flk-1 receptor and a protein kinase $\mathrm{C}$ signaling pathway. J. Biol. Chem. 1999; 274:33057-33063. [PubMed: 10551875]

21. Pathak AK, Bhutani M, Nair AS, Ahn KS, Chakraborty A, Kadara H, Guha S, Sethi G, Aggarwal BB. Ursolic acid inhibits STAT3 activation pathway leading to suppression of proliferation and chemosensitization of human multiple myeloma cells. Mol. Cancer Res. 2007; 5:943-955. [PubMed: 17855663]

22. Daigle I, Yousefi S, Colonna M, Green DR, Simon HU. Death receptors bind SHP-1 and block cytokine-induced anti-apoptotic signaling in neutrophils. Nat. Med. 2002; 8:61-67. [PubMed: 11786908]

23. Lucas R, Garcia I, Donati YR, Hribar M, Mandriota SJ, Giroud C, Buurman WA, Fransen L, Suter PM, Nunez G, Pepper MS, Grau GE. Both TNF receptors are required for direct TNF-mediated cytotoxicity in microvascular endothelial cells. Eur. J. Immunol. 1998; 28:3577-3586. [PubMed: 9842900]

24. Aragonés J, Schneider M, Van Geyte K, Fraisl P, Dresselaers T, Mazzone M, Dirkx R, Zacchigna S, Lemieux H, Jeoung NH, Lambrechts D, Bishop T, Lafuste P, Diez-Juan A, Harten SK, Van Noten P, De Bock K, Willam C, Tjwa M, Grosfeld A, Navet R, Moons L, Vandendriessche T, Deroose C, Wijeyekoon B, Nuyts J, Jordan B, Silasi-Mansat R, Lupu F, Dewerchin M, Pugh C, Salmon P, Mortelmans L, Gallez B, Gorus F, Buyse J, Sluse F, Harris RA, Gnaiger E, Hespel P, Van Hecke P, Schuit F, Van Veldhoven P, Ratcliffe P, Baes M, Maxwell P, Carmeliet P. Deficiency or inhibition of oxygen sensor Phd1 induces hypoxia tolerance by reprogramming basal metabolism. Nat. Genet. 2008; 40:170-180. [PubMed: 18176562] 


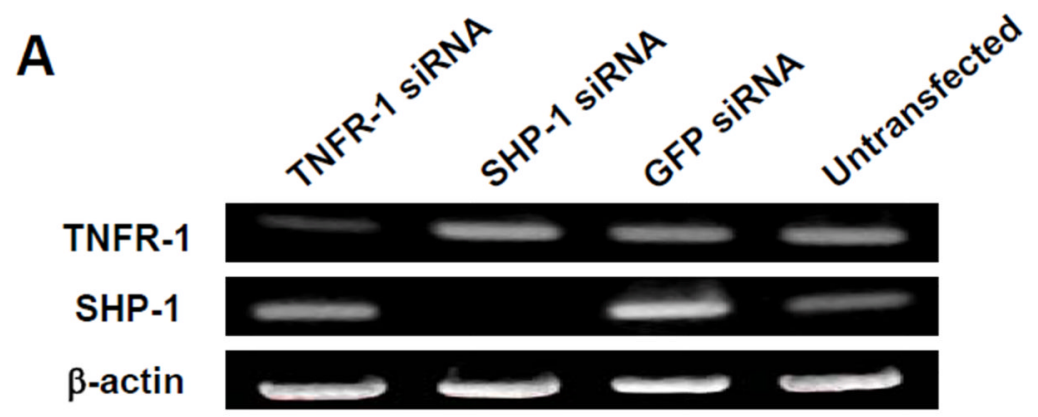

B

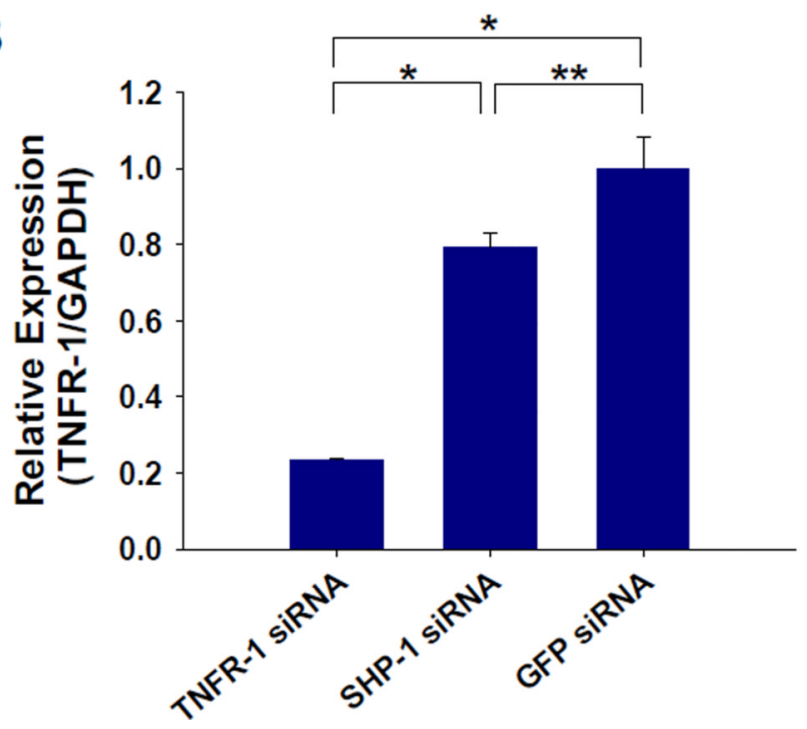

C

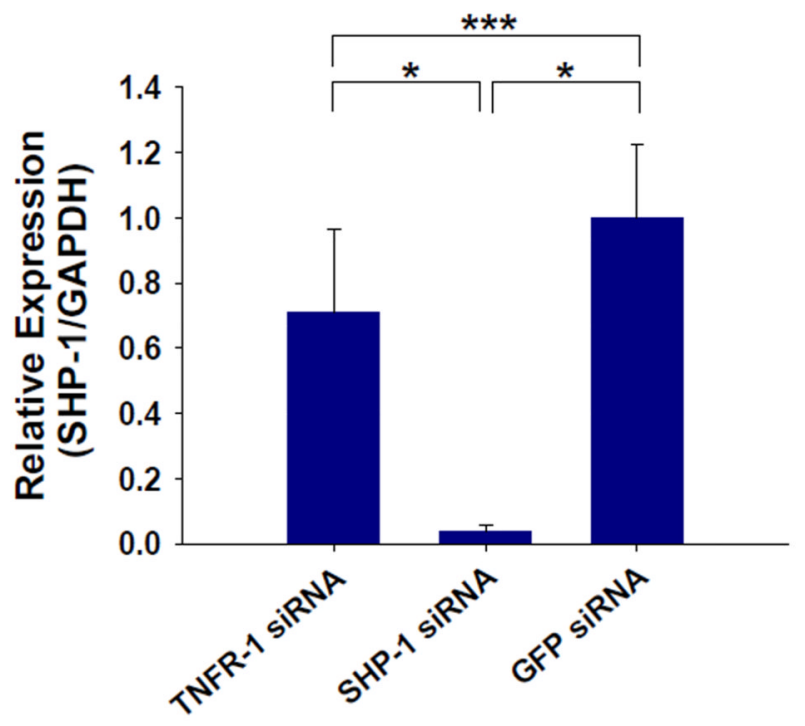

Fig. 1.

Silencing of target gene (TNFR-1 and SHP-1) expression by siRNA transfection. (A) RTPCR of HUVECs for each target at 2 days after transfection with TNFR-1, SHP-1, or GFP siRNA. Quantitative real-time PCR of siRNA-transfected HUVECs $(n=3)$ for (B) TNFR-1 and (C) SHP-1. The each gene expression in TNFR-1 or SHP-1 siRNA-transfected HUVECs was normalized to that in GFP siRNA-transfected HUVECs. TNFR-1 and SHP-1 siRNA delivery using Lipofectamine 2000 resulted in a significant and specific silencing of each molecule's expression compared with GFP siRNA delivery (*; $\mathrm{p}<0.01,{ }^{* *} ; \mathrm{p}<0.05$, $* * * ; \mathrm{p}>0.05)$. 
A

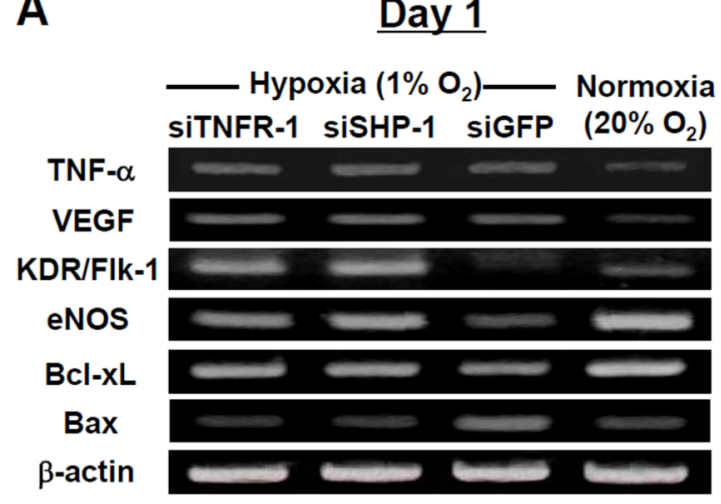

\section{Day 2}

Hypoxia $\left(1 \% \mathrm{O}_{2}\right)$ - Normoxia SITNFR-1 SISHP-1 SiGFP $\left(20 \% \mathrm{O}_{2}\right)$

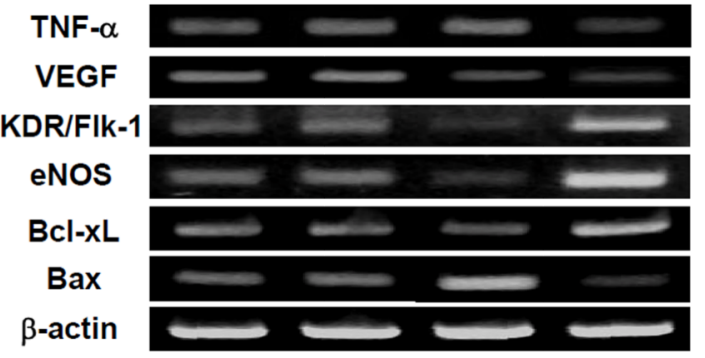

B

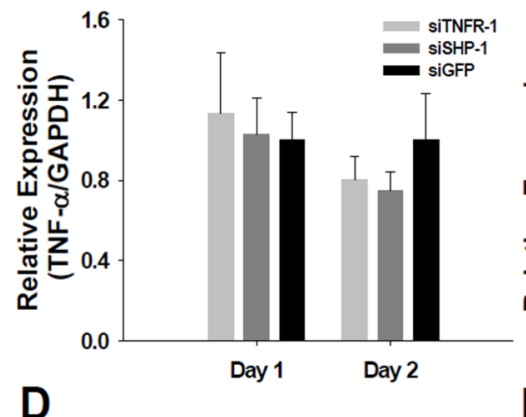

D 6 siTNRR-1

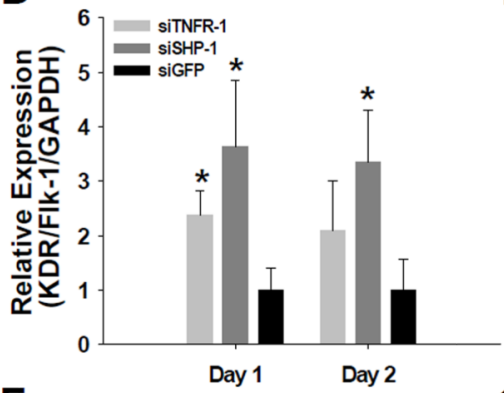

F

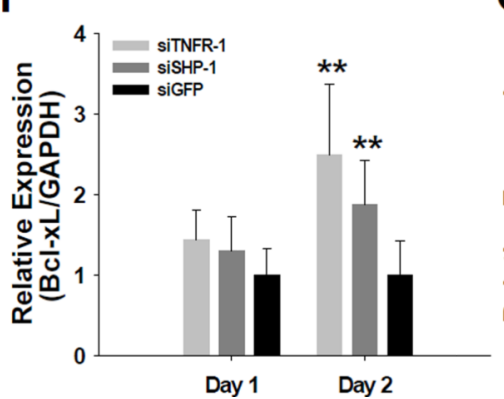

C

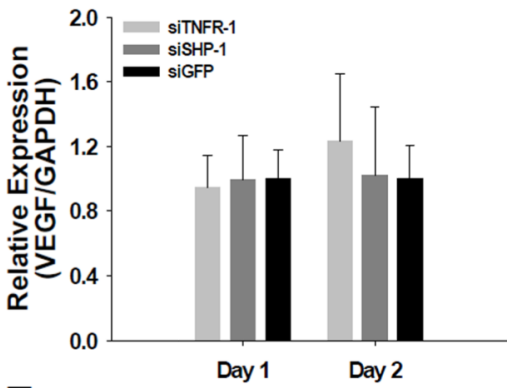

E

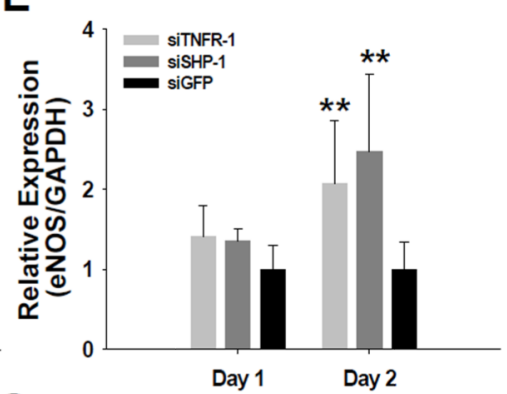

G

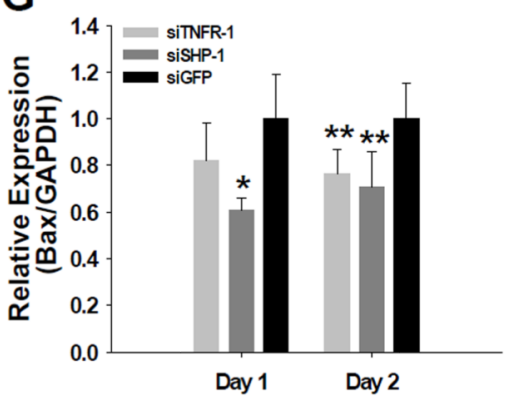

Fig. 2.

Gene expression profiles in siRNA-transfected HUVECs under hypoxic (1\% oxygen) and survival factor-deprived (EBM-2 basal medium with no serum and growth factors) conditions. (A) RT-PCR for various molecules of siRNA-transfected HUVECs retrieved at days 1 and 2 after culture. Quantitative real-time PCR for (B) TNF-a, (C) VEGF, (D) KDR/ Flk-1, (E) eNOS, (F) Bcl-xL, and (G) Bax. The gene expression levels of TNFR-1 or SHP-1 siRNA-transfected HUVECs $(n=4)$ at days 1 and 2 was normalized to that in GFP siRNAtransfected HUVECs $(* ; p<0.01, * * ; p<0.05$, compared to GFP siRNA group). 
A

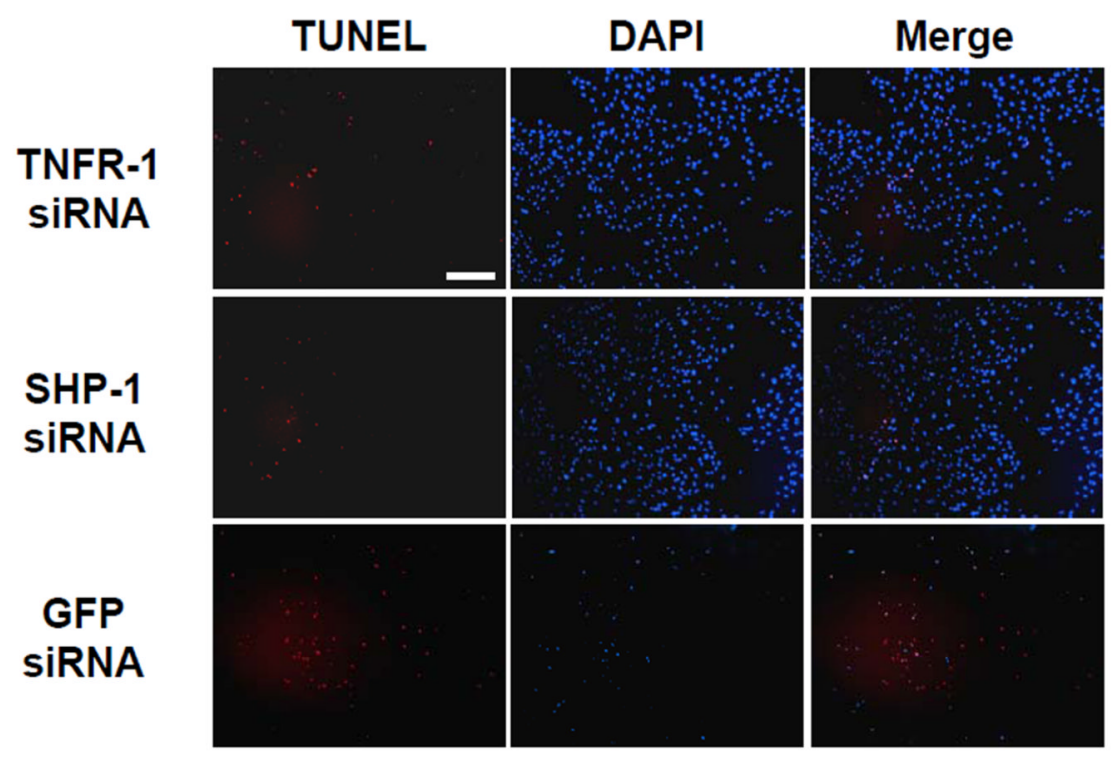

B

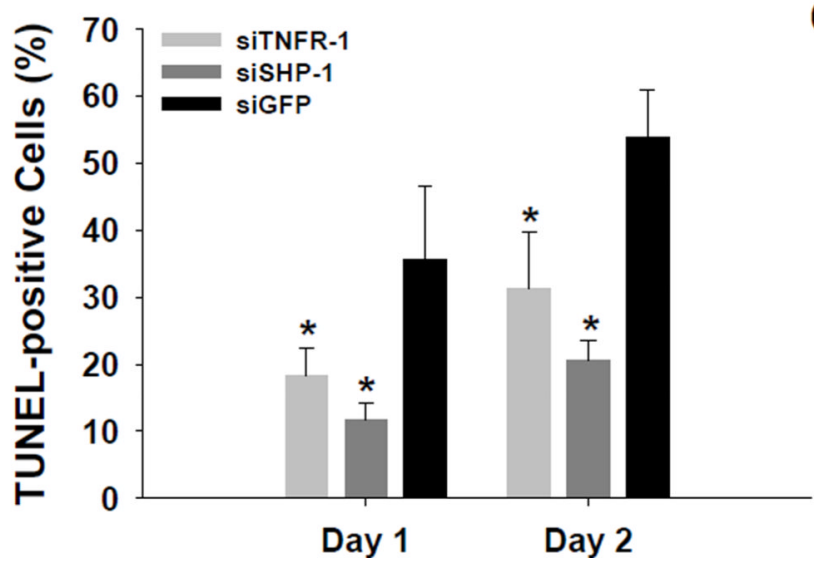

C

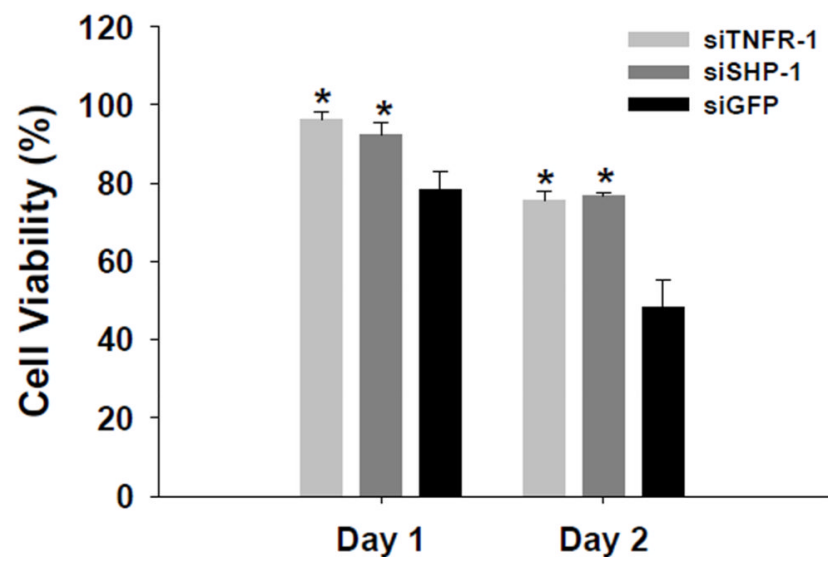

Fig. 3.

Apoptotic activity of siRNA-transfected HUVECs cultured under hypoxic (1\% oxygen) and survival factor-deprived (EBM-2 basal medium with no serum and growth factors) conditions. (A) TUNEL staining of siRNA-transfected HUVECs at 2 days after culture ( $\times 100)$. Scale bar indicates $200 \mu \mathrm{m}$. (B) The percentage ratio of TUNEL-positive cells to total cells at days 1 and 2 after culture ( $n=4, * ; p<0.01$, compared to GFP siRNA group). (C) The viability of siRNA-transfected HUVECs at days 1 and 2 after culture $(n=4, * ; p<0.01$, compared to GFP siRNA group). 
TNFR-1 siRNA

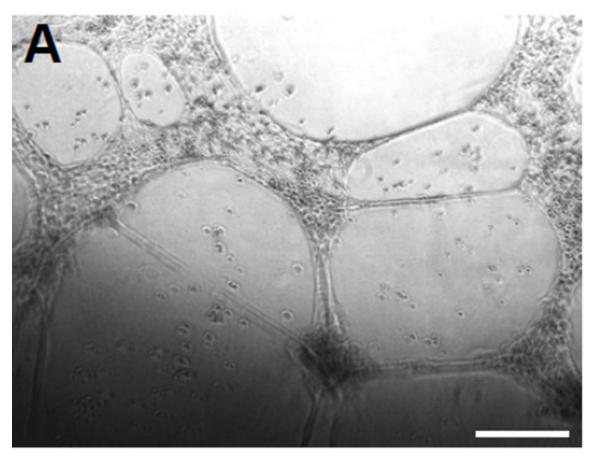

D

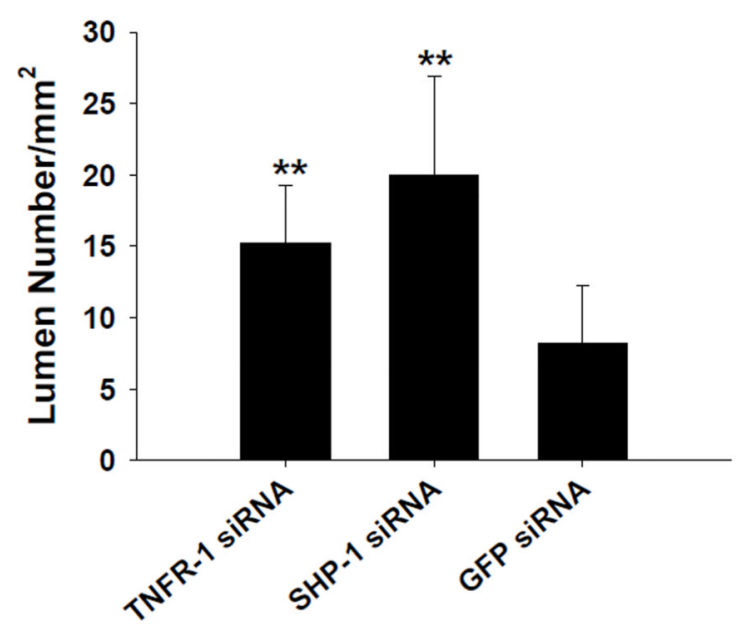

SHP-1 SiRNA

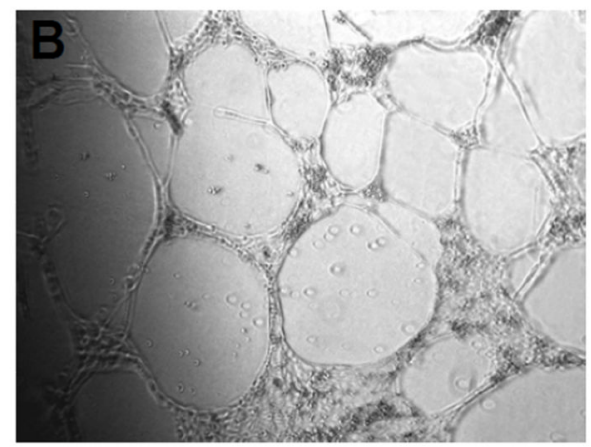

E

Fig. 4.

Capillary formation by siRNA-transfected HUVECs cultured for 2 days under hypoxic (1\% oxygen) and survival factor-deprived (EBM-2 basal medium with no serum and growth factors) conditions. When cultured onto GFR-Matrigel, HUVECs transfected with siRNAs for (A) TNFR-1 and (B) SHP-1 showed more extensive capillary structure formation, compared to HUVECs transfected with (C) GFP siRNA (×100). Scale bar indicates $200 \mu \mathrm{m}$. The density of (D) lumens and (E) tubes in the vascular structures generated on GFRMatrigel (*; $\mathrm{p}<0.01, * * ; \mathrm{p}<0.05$, compared to GFP-siRNA group). 
Table 1

Primer sequences and PCR product sizes for target genes

\begin{tabular}{|c|c|c|}
\hline Gene & Primer sequences (sense and anti-sense) & Product size \\
\hline \multirow{2}{*}{ TNFR-1 } & $5^{\prime}$-ACC AAG TGC CAC AAA GGA AC- $3^{\prime}$ & \multirow{2}{*}{263 bp } \\
\hline & $5^{\prime}$-CTG CAA TTG AAG CAC TGG AA- $3^{\prime}$ & \\
\hline \multirow{2}{*}{ SHP-1 } & 5'-GGC TTC TGG GAG GAG TTT GAG-3' & \multirow{2}{*}{452 bp } \\
\hline & $5^{\prime}$-CGG AGT TTG TAT TCG GTT GTG-3' & \\
\hline \multirow{2}{*}{ KDR/Flk-1 } & 5'-CAA CAA GCG GAG AGG AG-3' & \multirow{2}{*}{$587 \mathrm{bp}$} \\
\hline & $5^{\prime}$-ATG ACG ATG GAC AAG TAC CC- $3^{\prime}$ & \\
\hline \multirow{2}{*}{ eNOS } & 5'-GTG ATG GCG AAG CGA GTG AA--3' & \multirow{2}{*}{$421 \mathrm{bp}$} \\
\hline & $5^{\prime}$-CCG AGC CCG AAC ACA CAG AA- $3^{\prime}$ & \\
\hline \multirow{2}{*}{ VEGF } & 5'-CTT GCC TTG CTG CTC TAC CT-3' & \multirow{2}{*}{$484 \mathrm{bp}$} \\
\hline & $5^{\prime}$-CCT TGC AAC GCG AGT CTG T- $3^{\prime}$ & \\
\hline \multirow{2}{*}{ TNF-a } & 5'-ATG AGC ACT GAA AGC ATG ATC-3' & \multirow{2}{*}{700 bp } \\
\hline & 5'-TCA CAG GGC AAT GAT CCC AAA GTA GAC CTG CCC-3' & \\
\hline \multirow{2}{*}{ Bcl-xL } & $5^{\prime}$-TTG GAC AAT GGA CTG GTT GA- $3^{\prime}$ & \multirow{2}{*}{$780 \mathrm{bp}$} \\
\hline & $5^{\prime}$-GTA GAG TGG ATG GTC AGT G-3' & \\
\hline \multirow{2}{*}{ Bax } & $5^{\prime}$-TGG CAG CTG ACA TGT TTT CTG AC-3' & \multirow{2}{*}{$204 \mathrm{bp}$} \\
\hline & 5'-TCA CCC АAC CAC CCT GGT CTT- $3^{\prime}$ & \\
\hline \multirow{2}{*}{$\beta$-actin } & 5'-ATC TGG CAC CAC ACC TTC TA- $3^{\prime}$ & \multirow{2}{*}{$838 \mathrm{bp}$} \\
\hline & 5'-CGT CAT АCT CCT GCT TGC TG-3' & \\
\hline
\end{tabular}

Biochem Biophys Res Commun. Author manuscript; available in PMC 2014 March 18. 